\title{
Human Robot Interaction in Mobile Robot Applications
}

\author{
Akihisa Ohya \\ PRESTO, JST / University of Tsukuba \\ 1-1-1 Tennodai, Tsukuba 305-8573, Japan \\ E-mail ohya@is.tsukuba.ac.jp
}

\begin{abstract}
We want to reveal the usefulness of mobile robots by showing concrete applications in human daily life through this study. In this paper, our trials to realize several specific tasks are shown. The development of an Intelligent Escort Robot moving together with human is presented first. A teleoperated book browsing robot is described next. And finally, a network-based stationeries rental service performed by an autonomous mobile robot is introduced. We present the effectiveness of our approaches to the task realization by showing the experimental results using real mobile robots.
\end{abstract}

\section{Introduction}

In recent years, mobile robots have become autonomous enough, so that we have to think of their applications. We are considering in this study how robots can render service by moving by themselves. For the past years, many applications of mobile robot have been developed and proposed. Most of them were made and served mainly for industrial purposes. Only a few mobile robot applications were designed and made with the purpose of supporting humans meaningfully or cooperating with them in order to accomplish a specific task in their daily life[1][2][3].

In order to spread the use of mobile robot in human daily life, there is an on-going need of researching not only in autonomy and navigation of mobile robots, but also in useful and impactful mobile robots applications helping us in our day-to-day tasks. For this purpose, interaction with humans is, beside autonomy, a key function for a robot taking part actively in human life. However, concerning applications of mobile robots in real life, their number and variety are limited and focused on delivery robots[4][5][6], guiding robots[7] and cleaning robots[8], etc.

Our aim is to expand the mobile robot applications to various useful tasks in our daily life by proposing possible applications and showing their usefulness through experiments using real mobile robots. In this paper, our trials to realize several specific tasks are shown. The development of an Intelligent Escort Robot moving together with human is presented first. It is able to support humans in everyday life by interacting with them. A teleoperated book brows-
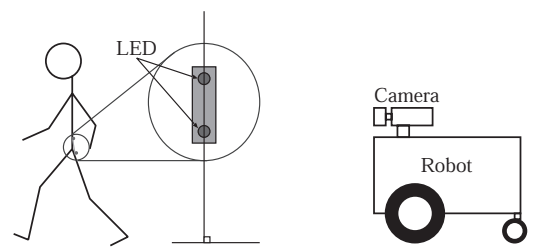

Figure 1: The escort robot detects the human having a device by using a camera.

ing robot is described next. Its task consists in perusing a book located in a remote place thanks to a robot. This robot can be considered as an access media that can interact physically with objects located in a remote place. And finally, a network-based stationeries rental service performed by an autonomous mobile robot is presented.

\section{Intelligent Escort Robot moving together with human}

The aim of this research is to develop an Intelligent Escort Robot moving along with people so that it can support them in everyday life by interacting with humans. Several concrete human helping robot applications can be considered. They are indoor and outdoor guidance and information supplying robots, accompanying or guiding people robots, or robots following humans while carrying heavy objects. In this section, the realization of a mobile robot system capable of following/accompanying people is described and some experimental results are shown.

\subsection{Human following behavior}

In order to follow a human, a mobile robot needs to know the position of the person and must be able to determine its own path in order to follow his target. In this study, we have equipped the person with a light- emitting device and made the robot detect this device using a camera as shown in Fig.1[9]. In order to appreciate the distance to the human, we use two LEDs fixed on a stick (Fig.2). The person carries this device perpendicular to the ground. Hence, by taking an image of this device, the robot is able to know the distance to the human thanks to the interval between the two lights in the image (Fig.3). It can also appreciate the direction taken by the device by determining 


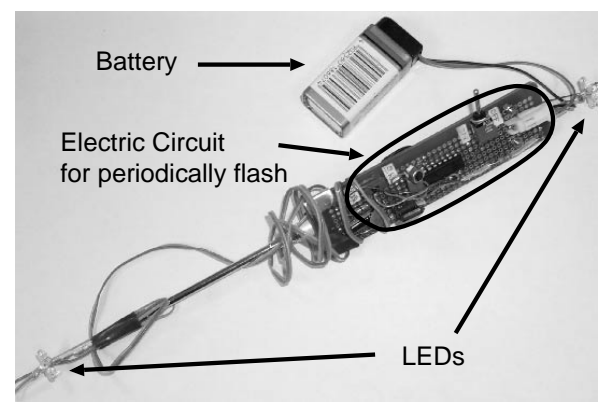

Figure 2: Light-emitting device used for recognizing human position in the experiments of the escort robot.

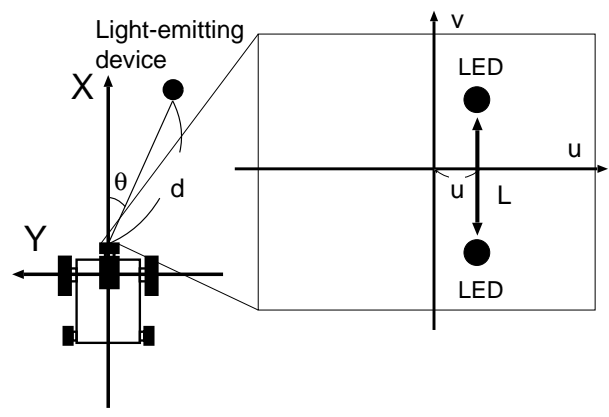

Figure 3: The schema of the image of LEDs obtained from camera mounted on the escort robot.

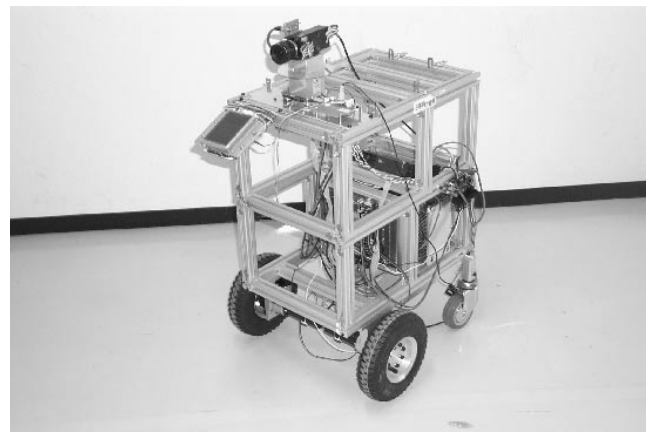

Figure 4: The mobile platform developed for the Intelligent Escort Robot Project.

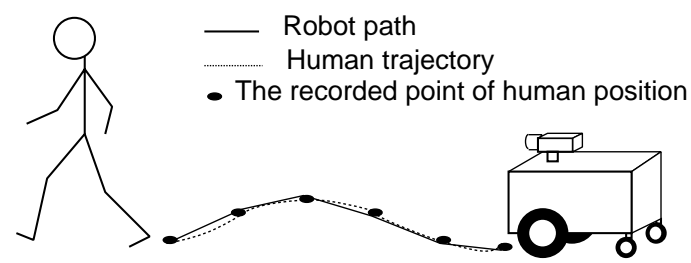

Figure 5: Determination of the path for human following. The positions of the human are recorded at a moderate interval.

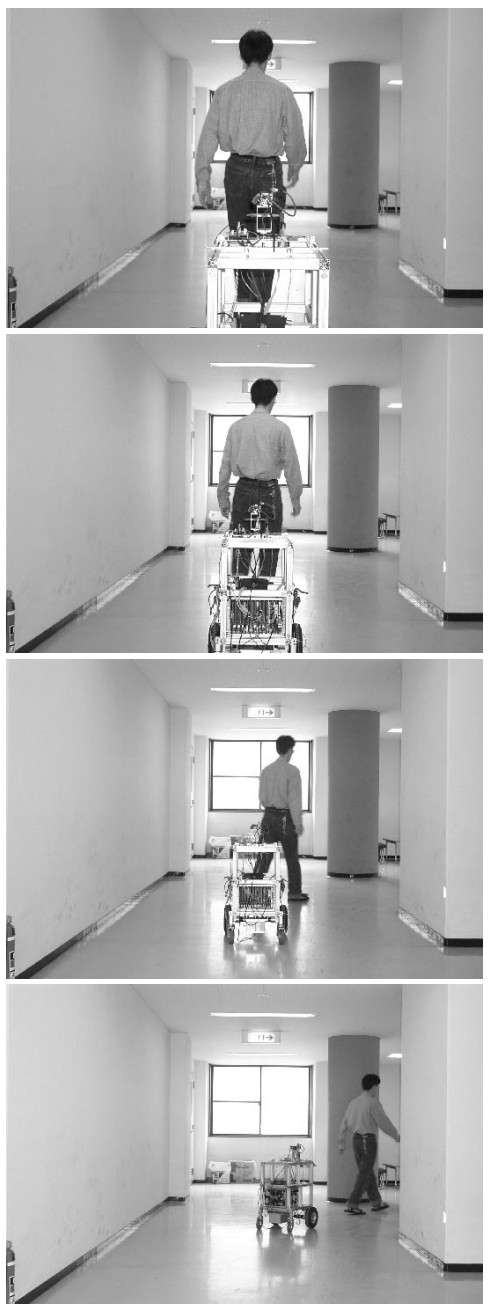

Figure 6: Experiment on the human following behavior of the escort robot.

the distance between the lights and the central vertical axis of the image and orienting the camera, thanks to a sensor stage having one degree of freedom (pan) installed on the top of the robot (Fig.4).

In order to prevent collision with obstacles, the robot should track the route followed by its target[10]. The robot calculates the distance between the previous and actual position of the human. If the human moved above a distance greater than a threshold value decided in advance, the position of the human is recorded in a list. Then, the robot reads each position recorded in the list successively and makes the appropriate movements to reach these positions (Fig.5). In order to estimate its position, the vehicle uses odometry and computes the location of the human relatively to the position of the vehicle. The location of the human is recorded by the robot in a global referential. The robot can adjust its speed according to the number of points recorded in the list that exist between the actual pose of the robot and the 


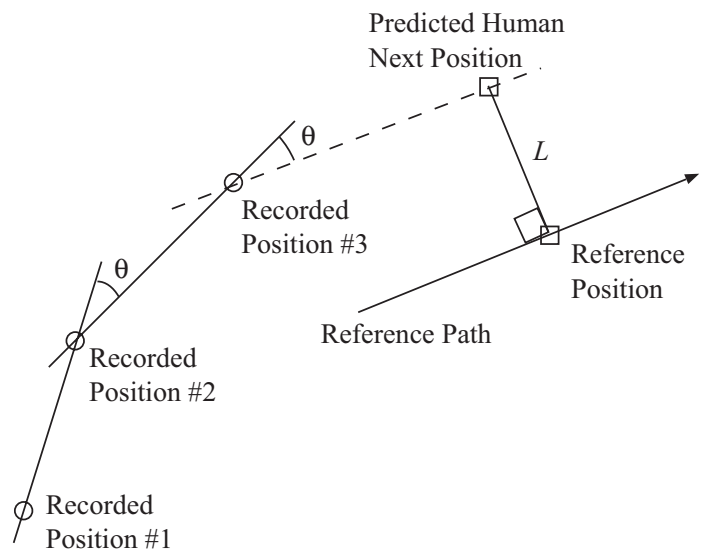

Figure 7: Prediction of the human next position and calculation of the reference position and the reference path for the accompanying behavior of the escort robot.

location of the human. Doing so enables the robot to track the path followed by its target. However, if an obstacle is found, the robot stops until it is removed.

Through experiments, the light-emitting device was carried by a person and the robot had to follow it. As obtained results, the robot could track the path followed by the target human. Figure 6 represents different steps of this experiment. The robot could easily distinguish his target in the environment existing multiple persons thanks to the device.

\subsection{Human accompanying behavior}

In order to accompany a person, the robot should estimate the next position of the person in order to move without any delay. The robot predicts the next position and speed of the person based on the history of its position with time recorded after every constant distance. The robot calculates the distance between the human's actual and previous recorded positions. If the human moved above a distance greater than a value decided in advance, the position of the human is recorded in a list. Assuming that the human will move with the same acceleration and the same angular velocity, the next human position can be calculated by linear approximation[11] (see Fig.7).

After predicting the next human position, the robot calculates the reference path and position where the robot should be in the next moment. The path is parallel to the predicted human's trajectory with a constant distance $L$ shown in Fig.7. The speed of the robot should be set so that the robot can reach the reference position on the next moment. Doing so enables the robot to run side by side with human by repeating these processes.

In order to prevent collision with obstacles, the robot should have an obstacle avoidance behavior. When the robot finds an obstacle on its path, it starts decreasing the distance between itself and the human. If the human per-

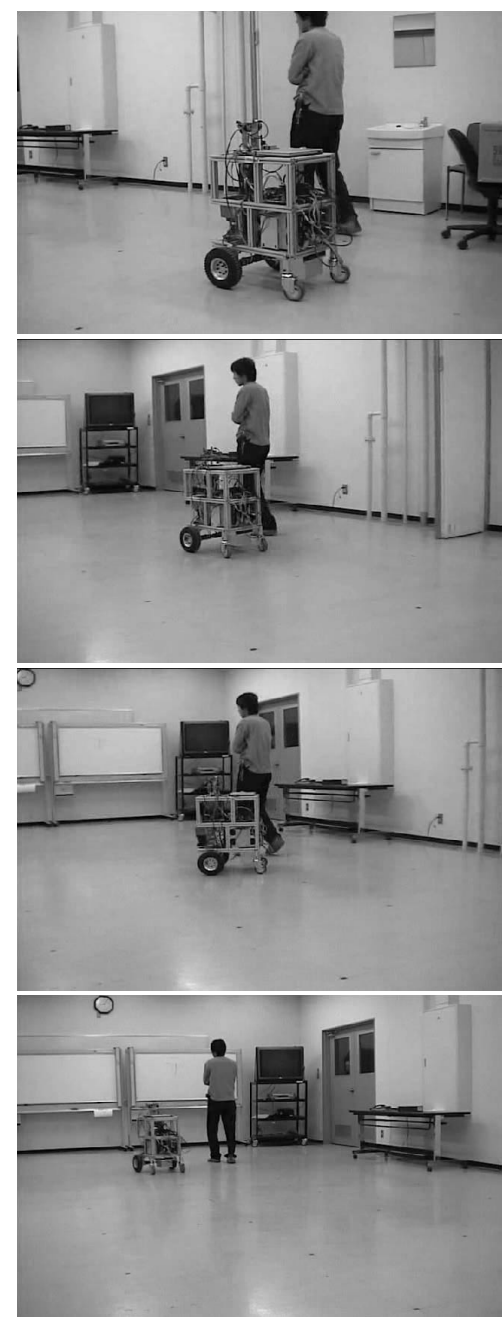

Figure 8: Experiment on the human accompanying behavior of the escort robot.

ceives this action and changes its path, the obstacle avoidance behavior may succeed fortunately. However, if the human doesn't change its path, the robot has to stop once and follow the human until the obstacle is completely avoided.

Experiments based on the method described above were conducted in an indoor room. The method using the lightemitting device mentioned in the previous section was used to detect the position of a person. As obtained results, the robot could accompany the person who carried the device while he was walking on a ' $S$ ' shaped path (Fig.8).

\section{Teleoperated book browsing robot}

This section describes a system which uses a mobile robot as a teleoperated tool for accessing and manipulating remote objects. The purpose of this work is to develop a robot system which helps humans to accomplish remotely a given task in their daily life, based on simple communication and mutual cooperation between them and 


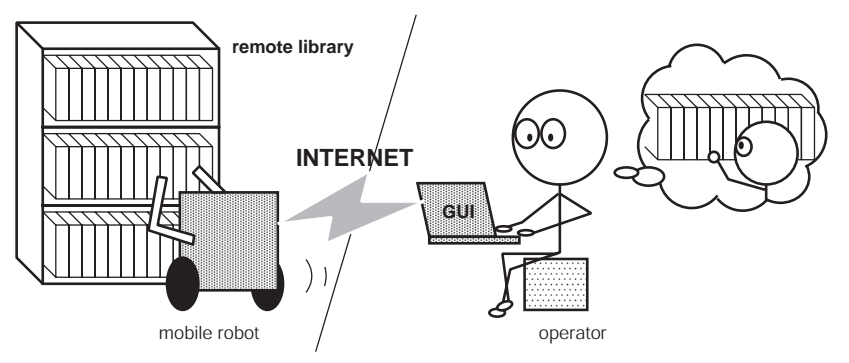

Figure 9: Concept of remote book browse using a mobile robot.

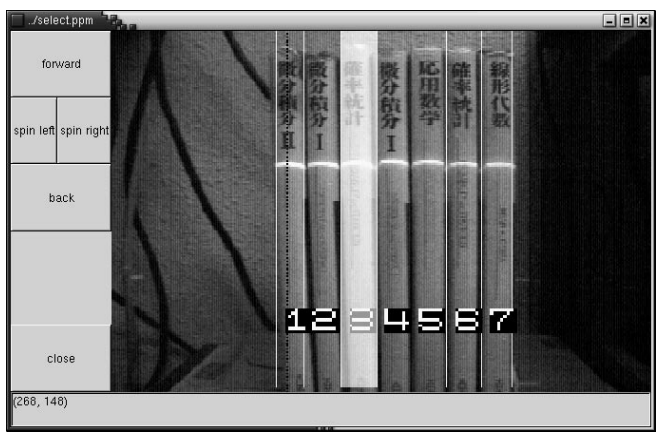

Figure 10: Displayed image in book selection mode.

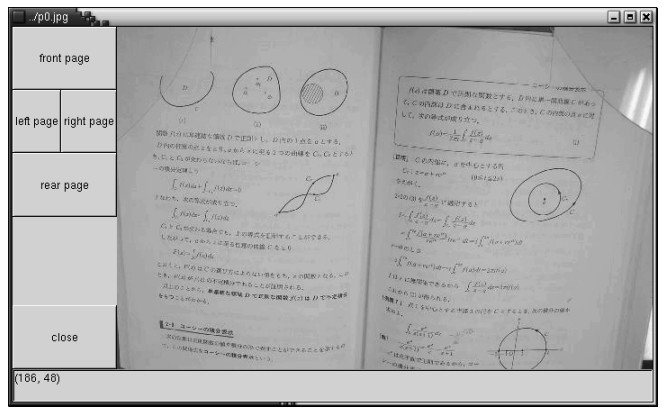

Figure 11: Displayed image in browsing mode.

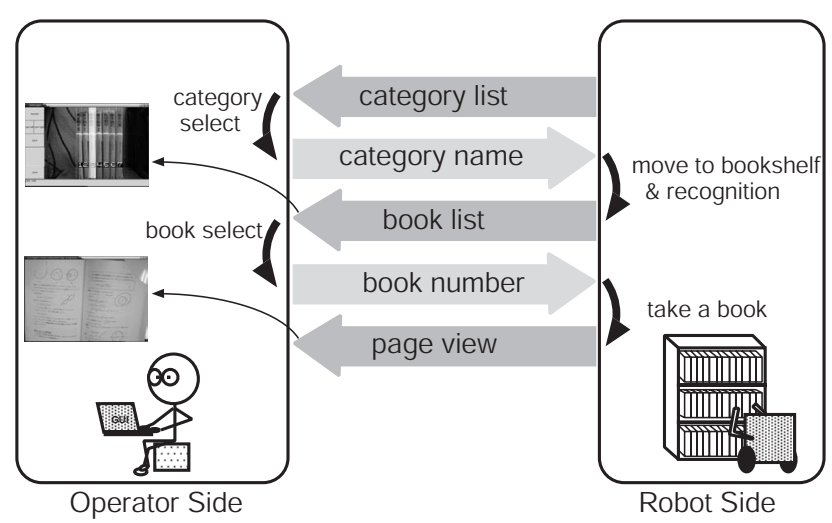

Figure 12: Flow of book browsing via teleoperated mobile robot.

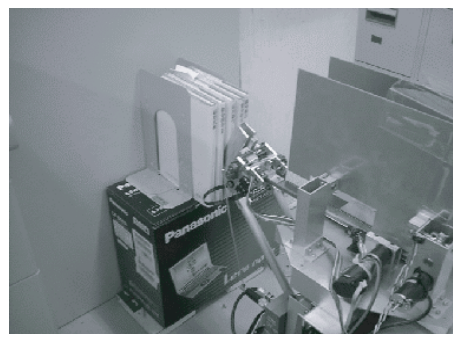

(a) approach to a book

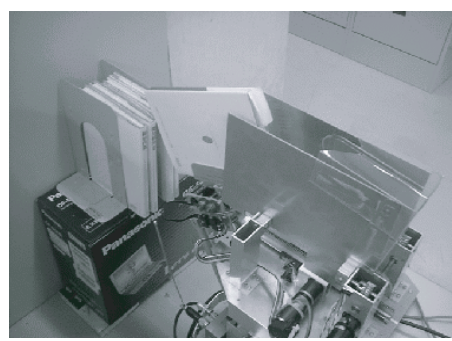

(b) extraction from bookshelf

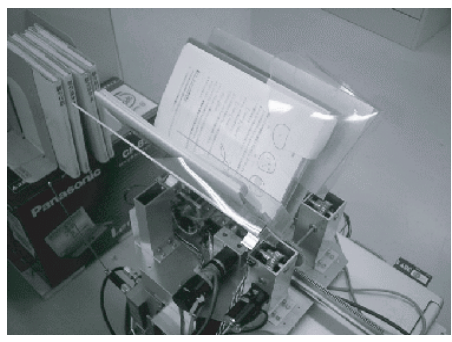

(c) book opening

Figure 13: Motion of the book browsing robot.

a teleoperated mobile robot. The specific task we set up in this research is a mobile robot which browses books from a library for remotely located humans. The concept of a remote books perusal is illustrated in Fig.9.

The list of books' categories in a library is provided by the robot to an operator. The operator searches and selects the book he wants to read from the categories list, and the robot moves toward the target bookshelf by generating a route autonomously and avoiding obstacles. This teleoperation interface is designed based on a basic strategy, which consists in specifying easily appropriate robot motions by selecting an object in an image displayed on the PC, and clicking buttons.

The interface for "book selection mode" is shown in Fig.10. The robot which arrived in front of the bookshelf looks for the position and boundary of each book by using a range sensor, and at the same time takes an image of the bookshelf sent to the operator. The boundary of each book is drawn on the taken image, and each book area is marked. Furthermore, after labeling each area with a number displayed on the screen, the user can select a book by 
choosing its number. The interface in "browsing mode" is shown in Fig.11. In the main screen, the zoomed image of an opened page is shown. If the page turning- over button located on the left of the screen is pushed, the page turningover equipment will then operate and the main screen will update with the picture of the following page.

The system we developed was tested to look at various books located in a specific place. The experiment flow is shown in Fig.12.

1. An operator accesses the robot through a network from his own PC, and selects the target category first.

2. When the robot reaches the bookshelf, it shifts to the book selection mode, and presents to the user the bookshelf image with labeled books.

3. The user chooses a book inside the window, and the robot lengthens its hand and picks up the book.

4. The perusal equipment is used to send to the user an image of a page of the manipulated book.

The robot motion steps from book extraction until page opening are shown in Fig.13. The book is re-shelved by the robot after perusal.

\section{Stationeries rental service robot}

In this section, a practical application of mobile robot playing an active part in human daily life is reported. The application introduced in this work is about a mobile robot operating in an office environment. The robot interacts and takes frequent orders from humans via a computer network and then navigates through a real world environment to provide them stationeries as items to rent and later on to return back. The stationeries are possessed and managed by the robot, in its body. A stationeries rental service is defined as the following task.

1. A person $X$ specifies from the screen of her computer, a stationery as an item she immediately needs.

2. From a remote location, a mobile robot tooled with various stationeries inside a box gets that information through LAN and checks if the sought stationery exists inside the item box.

3. If "Yes", then the robot moves through the office till $\mathrm{X}$ location and rents the item to $\mathrm{X}$. If "No", which means that another person $\mathrm{Y}$ has rented it, the robot contacts $\mathrm{Y}$ through her computer screen inquiring if she is ready to return the item back to the robot.

4. Based on Y's answer, the robot either moves to Y location to get the item back and then visits $\mathrm{X}$ to rent her the item, or the robot just transmits to $\mathrm{X}$ that the item rental service can not be provided due to someone else still using the item sought.

5. In addition of the above tasks, the mobile robot also furnishes to inhabitants of the office environment updated information of all items rental status and delivers desired stationeries at an appointed time to humans who previously booked items for later use.

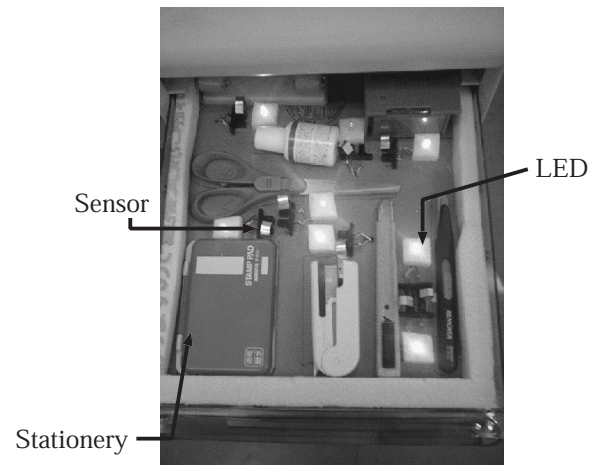

Figure 14: Stationeries stored in the item box.

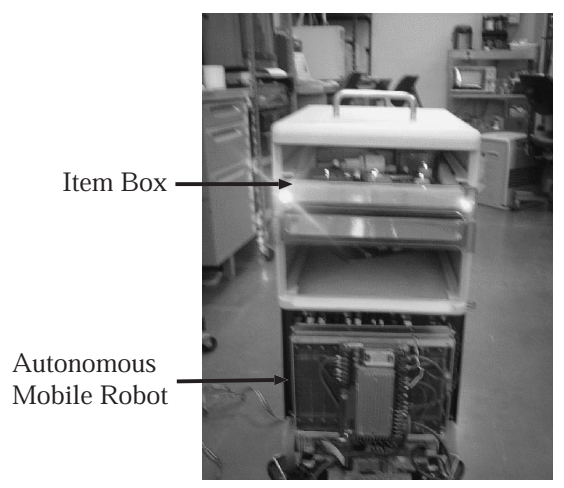

Figure 15: Autonomous mobile robot holding the item box.

As shown in Fig.14, we dispose a selection of stationeries in an item box drawers and set the box on the top of our mobile robot body (Fig.15). In order to enable our robot system to percept all stationeries existing inside the item box, we set appropriate sensors for each stationery.

We designed and developed a graphical user interface for human purpose (Fig.16). This interface is the access tool used by remote humans to interact with the robot system. It consists of three major panels, which are a service panel, a panel listing rental items and a message panel from the robot. For humans, to rent an item using this interface is an easy task going like, "First, check sequentially the items of your preference with the pointer device, then click lastly the desired service and view the robot answering to your request through the messaging panel." The local human interface is implemented as LED diodes set closely with every stationery and the item box drawers. The interface also includes voice synthesis.

The experiments were conducted in our laboratory environment. As illustrated sequentially in Fig.17, a remote human X -located a few meters from the robot-, requests an item (stapler) to the robot. The robot, next appreciates the feasibility of the requested service and then decides to navigate till $\mathrm{X}$ location and delivers the item. 


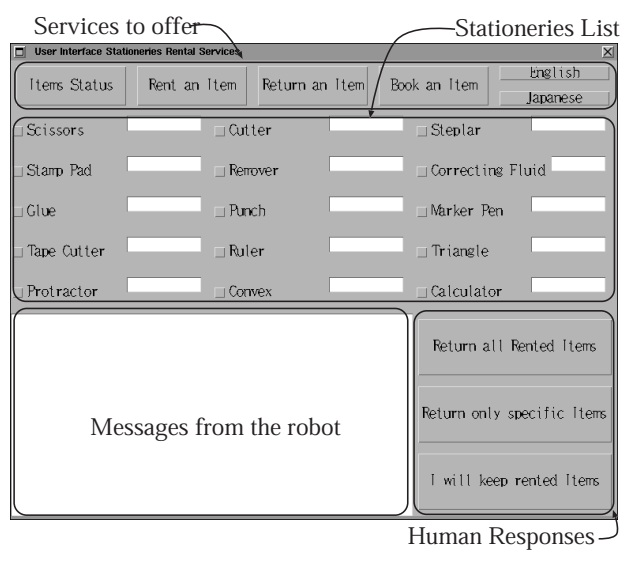

Figure 16: The human interface of the stationeries rental service robot system.

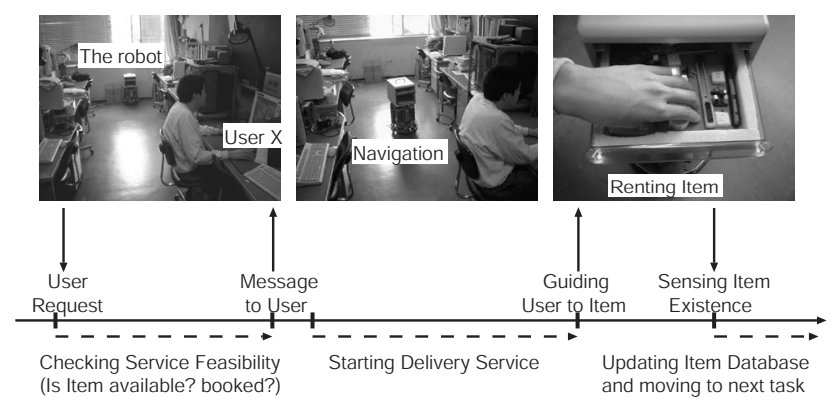

Figure 17: A sequential view of an item rental service.

\section{Conclusion}

In this paper, the several trials to study the usefulness of mobile robots are presented through different and concrete mobile robot applications. Each work must be completed and new application tasks should also be sought as future works. To act in our human daily life, the robot must have a function to interact with human of course. After realizing the function, their skills should be improved forwardly for a more comfortable human interaction. It is an important function and will be the next stage of the study on the human-robot interactive applications.

\section{Acknowledgements}

The author would like to express his gratitude to $\mathrm{Mr}$. Yousuke Nagumo, Mr. Tetsuo Tomizawa, Mr. Seydou Soumare and Mr. Takumi Munekata for their considerable contribution to the presented works done at Intelligent Robot Laboratory of University of Tsukuba.

\section{References}

[1] T. Tanaka, J. Ohwi, L. Litvintseva, K. Yamafuji, S. V. Ulyanov, I. Kurawaki: "A Mobile Robot for Service Use: Behavior Simulation system and Intelligent Control", 1997 IEEE/RSJ International Confer- ence on Intelligent Robots and Systems, pp. 366-372, 1997.

[2] Y. Hayashibara, Y. Sonoda, T. Takubo, H. Arai, K. Tanie: "Assist System for Carrying a Long Object with a Human -Analysis of a Human Cooperative Behavior n the Vertical Direction-", IEEE/RSJ International Conference on Intelligent Robots and Systems, pp. 695-700, 1999.

[3] R. Bischoff: "Advances in the Development of the Humanoid Service Robot HERMES", Field and Service Robotics Conference, pp. 156-161, 1999.

[4] J. M. Evans: "HelpMate: An autonomous mobile robot courier for hospitals", Proceedings of the International Conference on Intelligent Robots and Systems, pp. 1695-1700, 1994.

[5] R. Simmons, R. Goodwin, K. Z. Haigh, S. Koenig and J. O'Sullivan: "A layered architecture for office delivery robot", Proceedings of the 1st International Conference on Autonomous Agents, pp. 245-252, 1997.

[6] M. Hashima, F. Hasegawa, S. Kanda, T. Maruyama, T. Uchiyama:" Localization and Obstacle Detection for a Robot for Carrying Food Trays", Proceedings of the IEEE/RSJ International Conference on Intelligent Robots and Systems, pp. 345-351, 1997.

[7] S. Thurn, M. Bennewitz, W. Burgard, A. B. Cremers, F. Dellaert, D. Fox, D. Hahnel, C. Rosenberg, N. Roy, J. Shulte and D. Schulz: " MINERVA: A SecondGeneration Museum Tour-Guide Robot", Proceedings IEEE International Conference on Robotics and Automation, pp. 1999-2005, 1999.

[8] E. Prassler, E. Stroulia and M. Strobel: "Office Waste Cleanup: An Application for Service Robots", Proceedings IEEE International Conference on Robotics and Automation, pp. 1863-1868, 1997.

[9] Y. Nagumo and A. Ohya:"Human Following Behavior of an Autonomous Mobile Robot Using LightEmitting Device", Proceedings 10th IEEE International Workshop on Robot and Human Communication, pp. 225-230, 2001.

[10] M. Sato and K. Kosuge:"Handling of Object by Mobile Manipulator in Cooperation with Human Using Object Trajectory Following Method", IEEE/RSJ International Conference on Intelligent Robots and Systems, pp. 541-546, 2000.

[11] A. Ohya and T. Munekata: "Intelligent Escort Robot Moving together with Human -Interaction in Accompanying Behavior-", Proceedings 2002 FIRA Robot World Congress, pp. 31-35, 2002. 